\title{
Multidrug-Resistant Tuberculous Mediastinal Lymphadenitis, with an Esophagomediastinal Fistula, Mimicking an Esophageal Submucosal Tumor
}

\author{
Dongwuk Kim, Juwon Kim, Daegeun Lee, Ha Sung Chang, Hyunsung Joh, Won-Jung Koh and Jun Haeng Lee \\ Department of Medicine, Samsung Medical Center, Sungkyunkwan University School of Medicine, Seoul, Korea
}

Mediastinal tuberculous lymphadenitis rarely mimics esophageal submucosal tumor, particularly in the case of multidrug-resistant tuberculosis (MDR-TB). Herein, we report the case of a 61-year-old woman who visited a local hospital complaining of odynophagia. An initial esophagogastroduodenoscopy revealed an esophageal submucosal tumor, and subsequent chest computed tomography showed subcarinal lymphadenopathy with an esophagomediastinal fistula. The patient was then referred to Samsung Medical Center, and a second esophagogastroduodenoscopy showed deep central ulceration, as well as a suspicious fistula in the esophageal submucosal tumor-like lesion. A biopsy examination of the ulcerative lesion confirmed focal inflammation only. Next, an endobronchial, ultrasound-guided lymph node biopsy was performed, and TB was confirmed. The patient initially began a course of isoniazid, rifampicin, ethambutol, and pyrazinamide. However, after a drug sensitivity test, she was diagnosed with MDR-TB, and second-line anti-TB medications were prescribed. She recovered well subsequently. Clin Endosc 2016;49:564-569

Key Words: Tuberculosis; Lymphadenitis; Esophageal fistula

\section{INTRODUCTION}

Mediastinal tuberculous lymphadenitis is a kind of extrapulmonary tuberculosis (TB) that mainly involves the mediastinal lymph nodes. The disease is relatively rare in adults. ${ }^{1}$ Usually, the first abnormality to be detected is either a mediastinal mass or lymphadenopathy; common symptoms of TB, such as cough, fever, and weight loss, may occur subsequently. ${ }^{2}$ When mediastinal TB lymphadenitis involves the esophagus directly, it can cause esophageal symptoms like dysphagia or odynophagia. Indeed, one study from Japan reported esophageal symptoms in $17.6 \%(n=149)$ of patients

\footnotetext{
Received: January 5, 2016 Revised: March 29, 2016

Accepted: March 29, 2016

Correspondence: Jun Haeng Lee

Department of Medicine, Samsung Medical Center, Sungkyunkwan University School of Medicine, 81 Irwon-ro, Gangnam-gu, Seoul 06351, Korea

Tel: +82-2-3410-3409, Fax: +82-2-3410-6983, E-mail: stomachlee@gmail.com

(cc) This is an Open Access article distributed under the terms of the Creative Commons Attribution Non-Commercial License (http://creativecommons.org/ licenses/by-nc/3.0) which permits unrestricted non-commercial use, distribution, and reproduction in any medium, provided the original work is properly cited.
}

with mediastinal TB lymphadenitis. ${ }^{3}$

TB lymphadenitis mimicking an esophageal submucosal tumor is an uncommon finding, particularly at the first presentation of disease. Furthermore, although esophagomediastinal fistula can develop as a complication of this condition, it too is a rare complication. ${ }^{4}$ Herein, we report a case of multidrug-resistant (MDR)-TB mediastinal lymphadenitis, with esophagomediastinal fistula, which first mimicked an esophageal submucosal tumor.

\section{CASE REPORT}

A 61-year-old woman visited a local hospital complaining of odynophagia, lower neck pain, and upper anterior chest discomfort that had continued for about 10 days. At the time of presentation, the patient had not suffered fever, had night sweats, or weight loss. She had contracted cervical TB lymphadenitis at the age of 6 years, which was treated using anti-TB drugs.

The findings on an initial chest radiograph were normal; the 
patient then underwent an esophagogastroduodenoscopy that revealed a 1.5-cm esophageal submucosal tumor-like lesion 27 $\mathrm{cm}$ from the incisors (Fig. 1). On examining a biopsy specimen, chronic esophagitis was confirmed. Four weeks later, endoscopic ultrasonography was performed. Grossly, there was a mucosal defect on top of the submucosal tumor-like lesion. Sonography showed that the defect had an irregular margin, as well as mixed echogenicity (Fig. 2). Subsequent computed tomography (CT) of the chest revealed several enlarged lymph nodes in the subcarinal area (Fig. 3). The patient was referred to the gastroenterology department of our hospital for further evaluation and management.

At the first visit to our hospital, her blood pressure was 107/64 mm Hg, her pulse rate was 74 beats per minute, her respiratory rate was 20 breaths per minute, her body temperature was $36.3^{\circ} \mathrm{C}$, and her oxygen saturation was $98 \%$ with no oxygen support. We reviewed the chest CT results that had been performed at the local hospital and observed sub- carinal lymph node enlargement with esophagonodal fistula. Moreover, localized ground-glass opacity was seen at the right lower lobe. However, we did not suspect any lesions of malignancy, nor did we carry out another CT scan. We suspected that inflamed subcarinal lymph nodes (maybe due to TB) had compressed and infiltrated the esophagus, and that this may have mimicked an esophageal submucosal tumor. Chest radiographic findings were also normal at our hospital (Fig. 4).

We performed esophagogastroduodenoscopy again-6 weeks after the patient first presented at her local hospitaland found two small, fistula-like lesions $24 \mathrm{~cm}$ from the incisors, as well as a $0.5-\mathrm{cm}$ ulcer $29 \mathrm{~cm}$ from the incisors that was exuding a whitish discharge. Biopsies taken at the ulcer site showed only focal, active inflammation. We performed an endobronchial, ultrasound-guided, transbronchial needle aspiration (EBUS-TBNA), and on this basis diagnosed mediastinal TB lymphadenitis. Biopsy of the subcarinal lymph nodes showed chronic granulomatous inflammation, as well
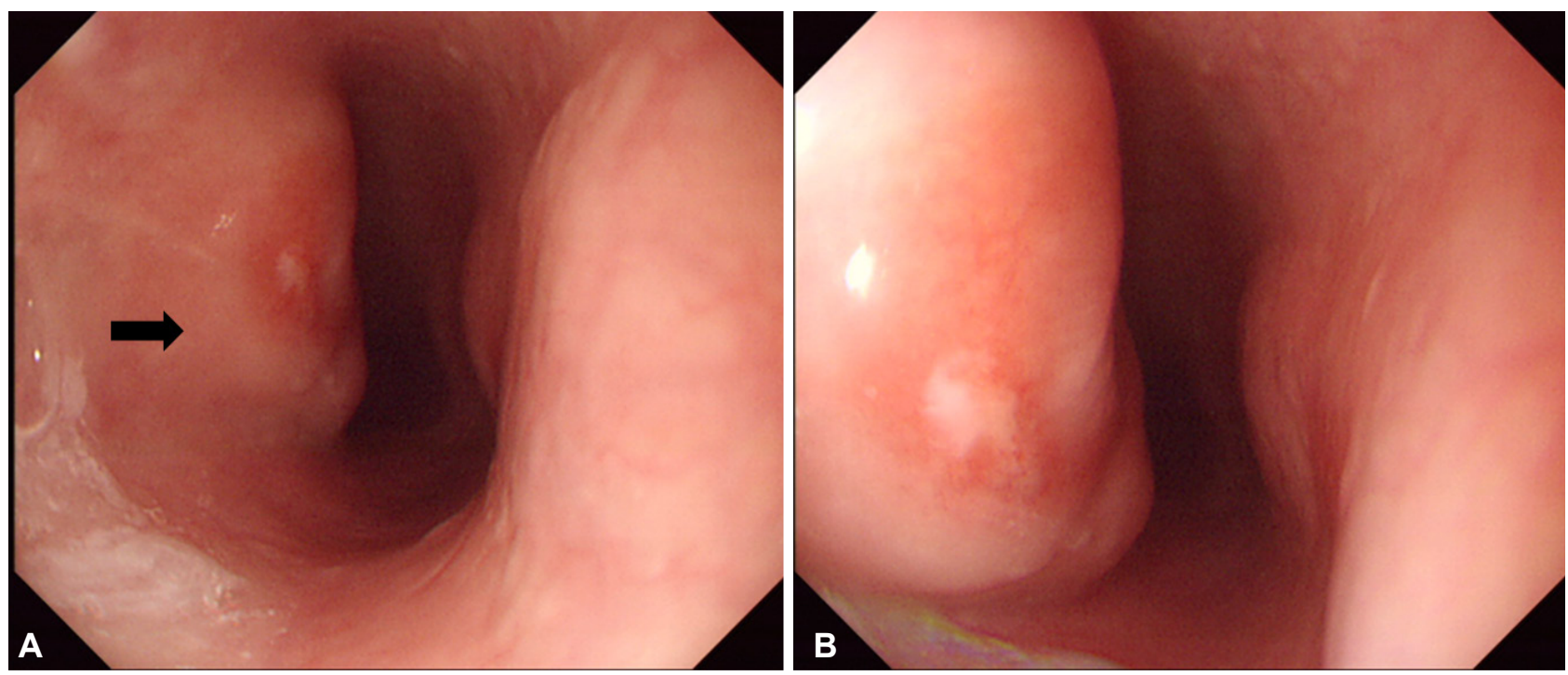

Fig. 1. (A, B) Initial esophagogastroduodenoscopic view of the esophageal submucosal tumor-like lesion (arrow) $27 \mathrm{~cm}$ from the upper incisor.
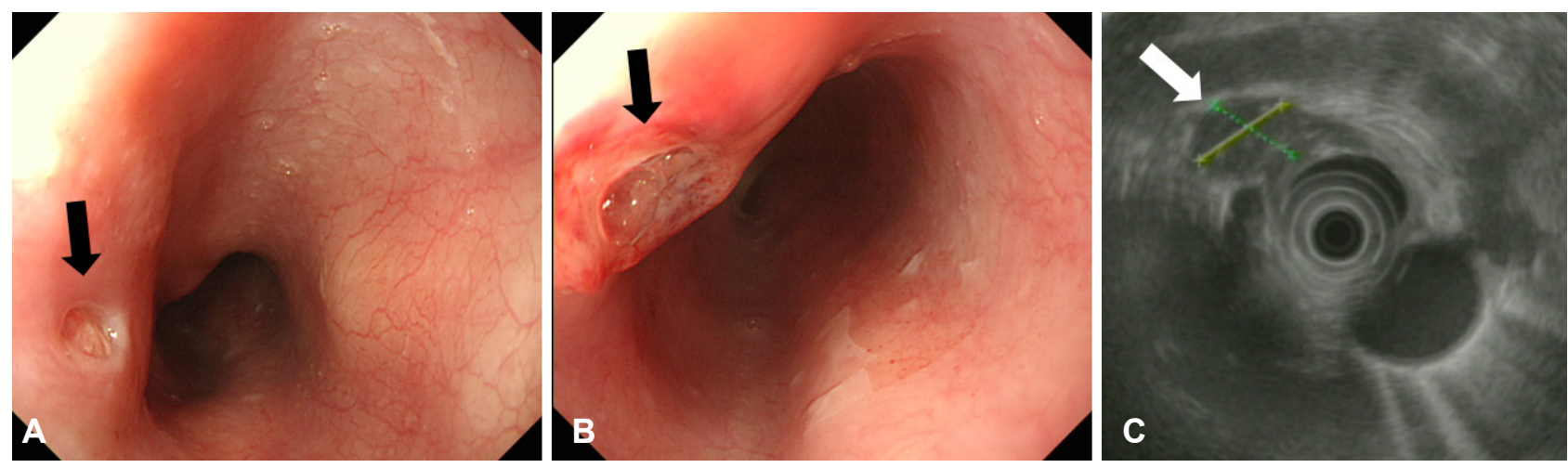

Fig. 2. Endoscopic ultrasonographic findings 4 weeks after the initial presentation. (A, B) A submucosal tumor-like lesion with ulceration (arrows) can be seen $27 \mathrm{~cm}$ from the upper incisors. (C) It shows mixed echogenicity and an irregular margin, and the esophageal wall layer cannot be distinguished on endoscopic ultrasonography. 


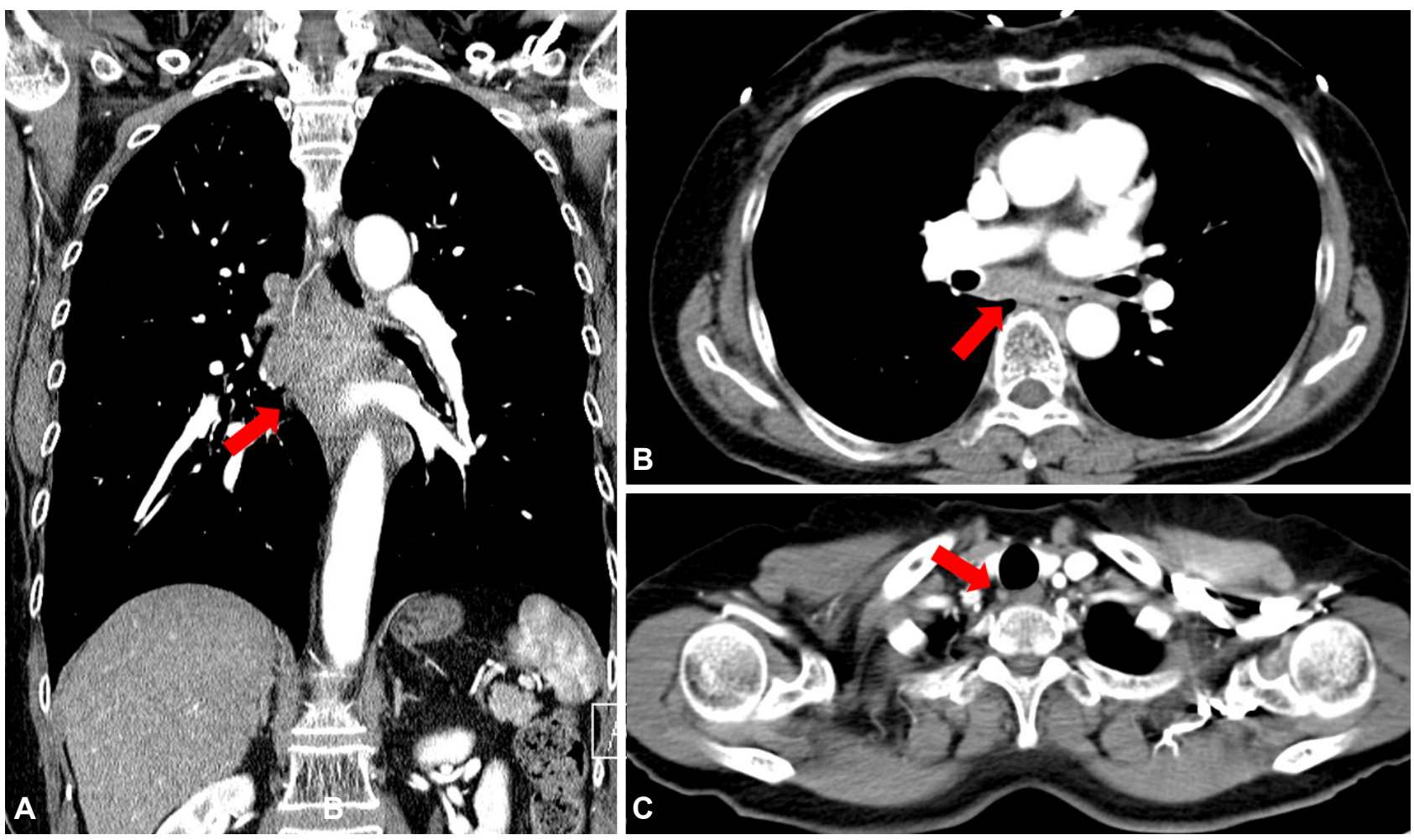

Fig. 3. (A, B) Chest contrast computed tomographic scan showing subcarinal lymphadenopathy with a probable fistulous connection to the esophageal lumen (arrows), (C) as well as paratracheal lymphadenopathy (arrow).

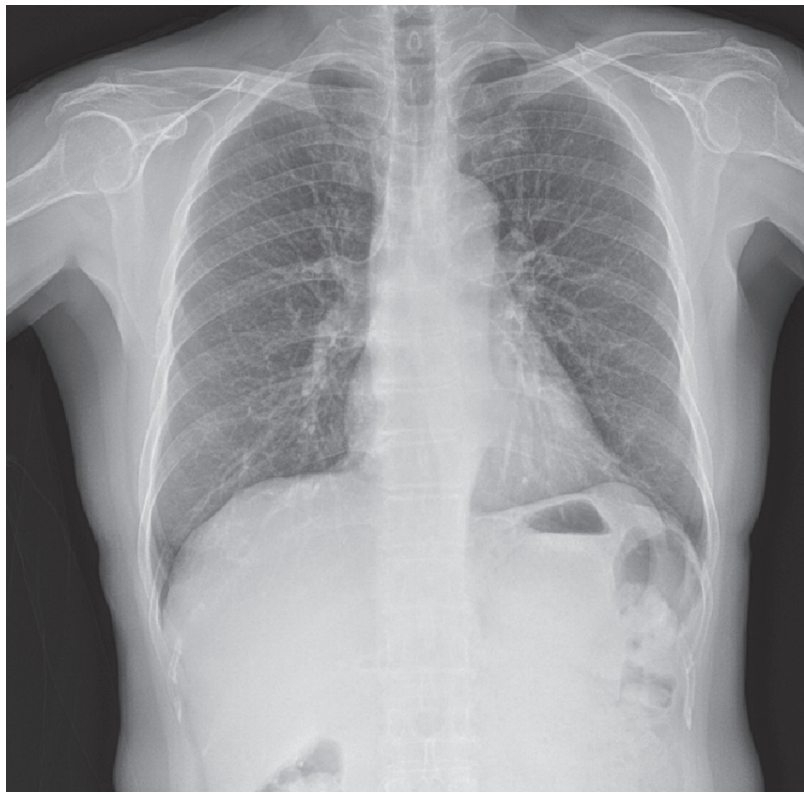

Fig. 4. An initial chest radiograph acquired at our hospital, showing normal chest findings.

as necrosis. Acid-fast bacilli (AFB) staining of the samples was negative, but a polymerase chain reaction for the TB $34 \mathrm{~K}$ protein chain reaction-based test for TB was positive. Hence, the patient was started on anti-TB treatment using isoniazid, rifampicin, ethambutol, pyrazinamide. Two weeks after the lymph node biopsy, Mycobacterium tuberculosis (M. tuberculosis) was isolated in a liquid-medium culture of the lymph node biopsy specimen. Considering the patient's history of $\mathrm{TB}$, drug resistance was a real possibility, and it was necessary to identify this early. To do so, we performed a reverse hybridization-based line probe assay, which revealed that the $M$. tuberculosis isolate harbored mutations in the $k a t G$ and $r p o B$ gene, indicating MDR-TB. More specifically, drug susceptibility testing (DST) using the mycobacteria growth-indicator tube in liquid culture demonstrated that the M. tuberculosis isolate was resistant to both isoniazid and rifampin. Hence, the patient was diagnosed with an MDR-TB infection, and the initial anti-TB drugs were discontinued 1 month after they had been prescribed. Second-line anti-TB drugs treatment was initiated; namely ethambutol, pyrazinamide, kanamycin, moxifloxacin, prothionamide, and cycloserine. Chest CT scanning performed at that time (Fig. 5) showed extensive lymphadenopathy with significant internal necrosis in the left supraclavicular, bilateral mediastinal, and intraabdominal areas. Active pulmonary TB was detected in the right lower lobe, as well as in the left upper lobe of the lungs. Chest posterioanterior radiograph showed mild ground-glass opacity in the left upper lobe.

Two months after the second-line anti-TB drugs had been 

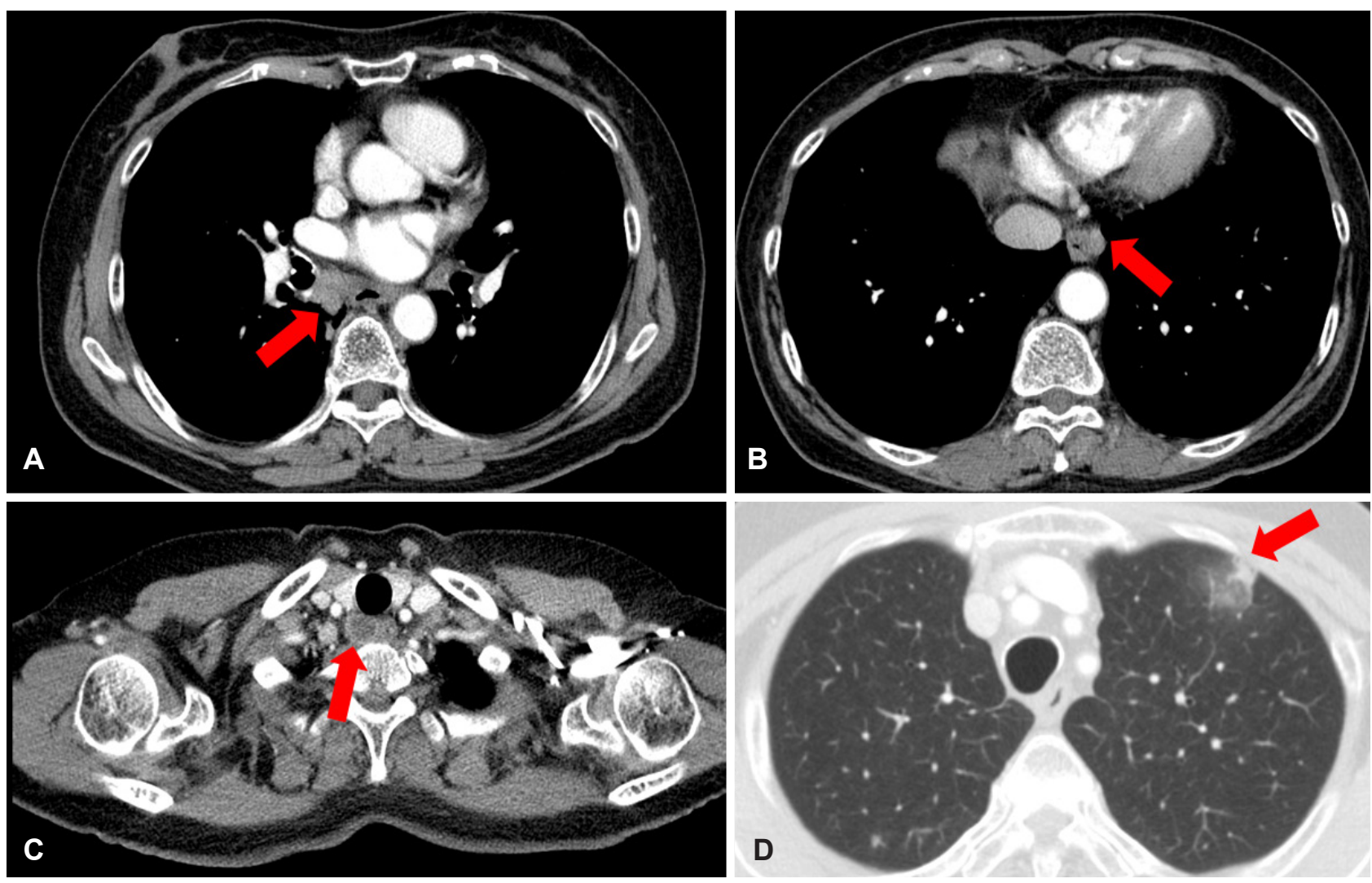

Fig. 5. Chest computed tomography scan 3 months after initial presentation. The extent of mediastinal lymphadenopathy is increased along the (A) subcarinal (arrow), (B) para-aortic (arrow), and (C) paratracheal lymph nodes (arrow), and (D) a new branching nodular lesion can be seen in the left upper lobe of the lung (arrow), which suggests pulmonary tuberculosis.

Table 1. Drug Sensitivity Test of Anti-Tuberculosis Drugs Using Lowenstein-Jensen Medium

\begin{tabular}{lccc}
\hline Drug name & Drug concentration, $\mathbf{~ c g} / \mathbf{m L}$ & Reading $^{\text {a) }}$ & Result \\
\hline Isoniazid & 0.2 & +++ & Resistant \\
Rifampin & 40 & +++ & Resistant \\
Streptomycin & 10 & +++ & Resistant \\
Ethambutol & 2.0 & ++ & Resistant \\
Kanamycin & 40 & - & Sensitive \\
Capreomycin & 40 & - & Sensitive \\
Prothionamide & 40 & - & Sensitive \\
Cycloserine & 30 & - & Sensitive \\
Para-amino salicylic acid & 1.0 & - & Sensitive \\
Ofloxacin & 2.0 & - & Sensitive \\
Moxifloxacin & 2.0 & - & Sensitive \\
Amikacin & 40 & - & Sensitive \\
\hline Levofloxacin & 2.0 & +++ & Sensitive \\
Rifabutin & 20 & Resistant \\
\hline
\end{tabular}

a) -, no isolated colonies; +, $\leq 100$ isolated colonies; ++, 101-200 isolated colonies; +++, 201-500 isolated colonies; ++++, more colonies than +++.

initiated, a follow-up chest CT scan showed improvement in the TB-related lymphadenopathy and pulmonary lesions. Conventional DST, using a solid culture medium, revealed that the M. tuberculosis isolate was resistant to ethambutol, streptomycin, and rifabutin, as well as to isoniazid and rifampin (Table 1). Hence, ethambutol was discontinued. After 4 
months of treatment, prothionamide was discontinued due to hypothyroidism, and after 6 months of treatment, kanamycin was discontinued. She was subsequently treated using pyrazinamide, moxifloxacin, and cycloserine for an additional 12 months. Esophagogastroduodenoscopy performed 3 months after the start of second-line treatment showed a normal esophagus. A follow-up chest CT scan obtained 6 months after the initiation of the second-line treatment showed neither mediastinal lymphadenopathy nor active lung lesions.

\section{DISCUSSION}

There have been some reports of mediastinal TB lymphadenitis secondary to pulmonary TB; nonetheless, cases of this disease that initially or solely involve the mediastinal lymph nodes are less common, accounting for a relatively small proportion of extrapulmonary TB cases. In one study conducted in 2009, extrapulmonary TB involved the lymph nodes in only $17.6 \%$ of cases. ${ }^{5}$ According to reports from both the United States and South Korea, the incidence of TB has decreased recently. ${ }^{6.7}$ However, this is largely due to a decrease in the incidence of pulmonary rather than that of extrapulmonary TB; the incidence of the latter is similar to what it was in the past, and it may even have risen slightly. Hence, the proportion of TB cases involving mediastinal TB lymphadenitis will probably increase. ${ }^{7}$

There have been several reports of esophageal TB or esophageal infiltration in mediastinal TB lymphadenitis mimicking an esophageal submucosal tumor. ${ }^{8-10}$ However, it is relatively rare for TB to present as an esophageal submucosal tumor upon the patient's first visit. In our case, subcarinal lymphadenopathy, caused by TB lymphadenitis, may have compressed and infiltrated the esophagus. We suspect that TB infiltration into the esophagus caused the formation of an esophagomediastinal fistula that mimicked a submucosal tumor. Hence, if an esophageal submucosal tumor-like lesion is detected, clinicians should consider the possibility that TB is mimicking a submucosal tumor, when making a diagnosis. When the patient has unusual symptoms, or TB is clinically suspected, further evaluation using chest CT or follow-up endoscopy can be helpful in this regard. It is difficult to distinguish a submucosal tumor from a fistula or extrinsic compression, using endoscopic ultrasonography alone.

Globally, $3.3 \%$ of the new cases, and 20\% of previously treated cases of TB involve MDR-TB. ${ }^{11}$ In South Korea, an analysis all patients with TB between 1994 and 2004 showed that the proportion of cases involving MDR-TB was 2.7\% among new cases and $14.0 \%$ among previously treated cases. ${ }^{12}$ In another study that assessed TB drug resistance at a private referral center in South Korea, the proportion of cases involving MDR-TB was 3.9\% among new cases and 27.2\% among previously treated cases. ${ }^{13}$ In our case, mediastinal lymphadenitis occurred as a result of MDR-TB. There have been occasional reports of similar disease processes, but the exact incidence is unknown. One study in South Korea, carried out in 2000, enrolled 373 cases of TB lymphadenitis. In only two cases $(0.5 \%)$ did TB lymphadenitis involve the mediastinal area lymph nodes alone. ${ }^{14}$

More recently, in 2014, a case of MDR-TB mediastinal lymphadenitis with esophagomediastinal fistula was reported. ${ }^{15}$ This case was similar to our own in several ways: both cases involved MDR-TB-induced mediastinal lymphadenitis and an esophagomediastinal fistula. However, in the 2014 case, esophagogastroduodenoscopy revealed only a fistula, and not a submucosal tumor. Furthermore, the diagnosis was not confirmed using EBUS-TBNA, and mediastinoscopy was performed. We also believe that our case was that of an early phase mediastinal TB lymphadenitis.

In patients with mediastinal TB lymphadenitis, invasive techniques, such as lymph node biopsy and subsequent culture, should be performed to ensure an accurate diagnosis. Previously, mediastinoscopy was the most important procedure in the diagnosis of mediastinal TB lymphadenitis, even though it is invasive. ${ }^{2}$ More recently, however, EBUS-TBNA technology has developed significantly, and it is now the most important tool. ${ }^{16}$ When diagnosis using EBUS-TBNA is not possible, mediastinoscopy or CT-guided biopsy can be helpful. Generally though, EBUS-TBNA is preferred, because other procedures are more invasive and difficult to perform. In our case, endoscopic ultrasonography-guided biopsy of the esophageal submucosal tumor-like lesion was another possible diagnostic technique. However, based on the esophagogastroduodenoscopy, endoscopic ultrasonography, and CT findings, we thought it would be easier to take tissue samples from the enlarged subcarinal lymph node using EBUS.

In our case, it was unclear whether the patient had early-phase pulmonary TB, and localized ground-glass opacity alone was found. Although after several months, lung lesions compatible with pulmonary TB could be visualized on chest CT. Therefore, we believe that imaging studies are more important in mediastinal lymphadenitis, and if patients with mediastinal lymphadenitis show pulmonary lesions, clinicians should strongly consider AFB staining and culture of sputum samples.

\section{Conflicts of Interest}

The authors have no financial conflicts of interest. 


\section{REFERENCES}

1. Amorosa JK, Smith PR, Cohen JR, Ramsey C, Lyons HA. Tuberculous mediastinal lymphadenitis in the adult. Radiology 1978;126:365-368.

2. Ayed AK, Behbehani NA. Diagnosis and treatment of isolated tuberculous mediastinal lymphadenopathy in adults. Eur J Surg 2001;167:334338.

3. Shiota Y, Kitade M, Ueda N, Furuya K. Tuberculous mediastinal lymphadenitis in an adult patient. Jpn J Med 1989;28:382-384.

4. Ohtake M, Saito H, Okuno M, Yamamoto S, Ohgimi T. Esophagomediastinal fistula as a complication of tuberculous mediastinal lymphadenitis. Intern Med 1996;35:984-986.

5. Lin JN, Lai CH, Chen $\mathrm{YH}$, et al. Risk factors for extra-pulmonary tuberculosis compared to pulmonary tuberculosis. Int J Tuberc Lung Dis 2009;13:620-625.

6. Kim JH, Yim JJ. Achievements in and challenges of tuberculosis control in South Korea. Emerg Infect Dis 2015;21:1913-1920.

7. Adada H, Valley MA, Nour SA, et al. Epidemiology of extra-pulmonary tuberculosis in the United States: high rates persist in the post-HIV era. Int J Tuberc Lung Dis 2014;18:1516-1521.

8. Mou Y, Zeng H, Wang QM, et al. Esophageal tuberculosis initially misdiagnosed by endoscopy as a submucosal tumor. Endoscopy 2015;47
Suppl 1 UCTN:E30-E31.

9. Kang MJ, Yi SY. Esophageal tuberculosis presenting as a submucosal tumor. Clin Gastroenterol Hepatol 2008;6:A26.

10. Huang YK, Wu YC, Liu YH, Liu HP. Esophageal tuberculosis mimicking submucosal tumor. Interact Cardiovasc Thorac Surg 2004;3:274-276.

11. World Health Organization. Global tuberculosis report 2015 [Internet]. Geneva: World Health Organization; c2016 [cited 2016 Apr 5]. Available from: http://www.who.int/tb/publications/global_report/en/.

12. Bai GH, Park YK, Choi YW, et al. Trend of anti-tuberculosis drug resistance in Korea, 1994-2004. Int J Tuberc Lung Dis 2007;11:571-576.

13. Choi JC, Lim SY, Suh GY, et al. Drug resistance rates of Mycobacterium tuberculosis at a private referral center in Korea. J Korean Med Sci 2007;22:677-681.

14. Park HJ, Ryoo HM, Shin KC, et al. A clinical study of tuberculous lymphadenitis. Tuberc Respir Dis 2000;48:730-739.

15. Ko Y, Lee HY, Lee YS, et al. Esophagomediastinal fistula secondary to multidrug-resistant tuberculous mediastinal lymphadenitis. Intern Med 2014;53:1819-1824.

16. Geake J, Hammerschlag G, Nguyen P, et al. Utility of EBUS-TBNA for diagnosis of mediastinal tuberculous lymphadenitis: a multicentre Australian experience. J Thorac Dis 2015;7:439-448. 